\title{
The unfolded protein response in human
}

skeletal muscle is not involved in the onset of glucose tolerance impairment induced by a fat-rich diet

\section{Louise Deldicque, Karen Van Proeyen, Marc Francaux \& Peter Hespel}

European Journal of Applied Physiology

ISSN 1439-6319

Volume 111

Number 7

Eur J Appl Physiol (2011)

111:1553-1558

DOI $10.1007 /$

s00421-010-1783-1

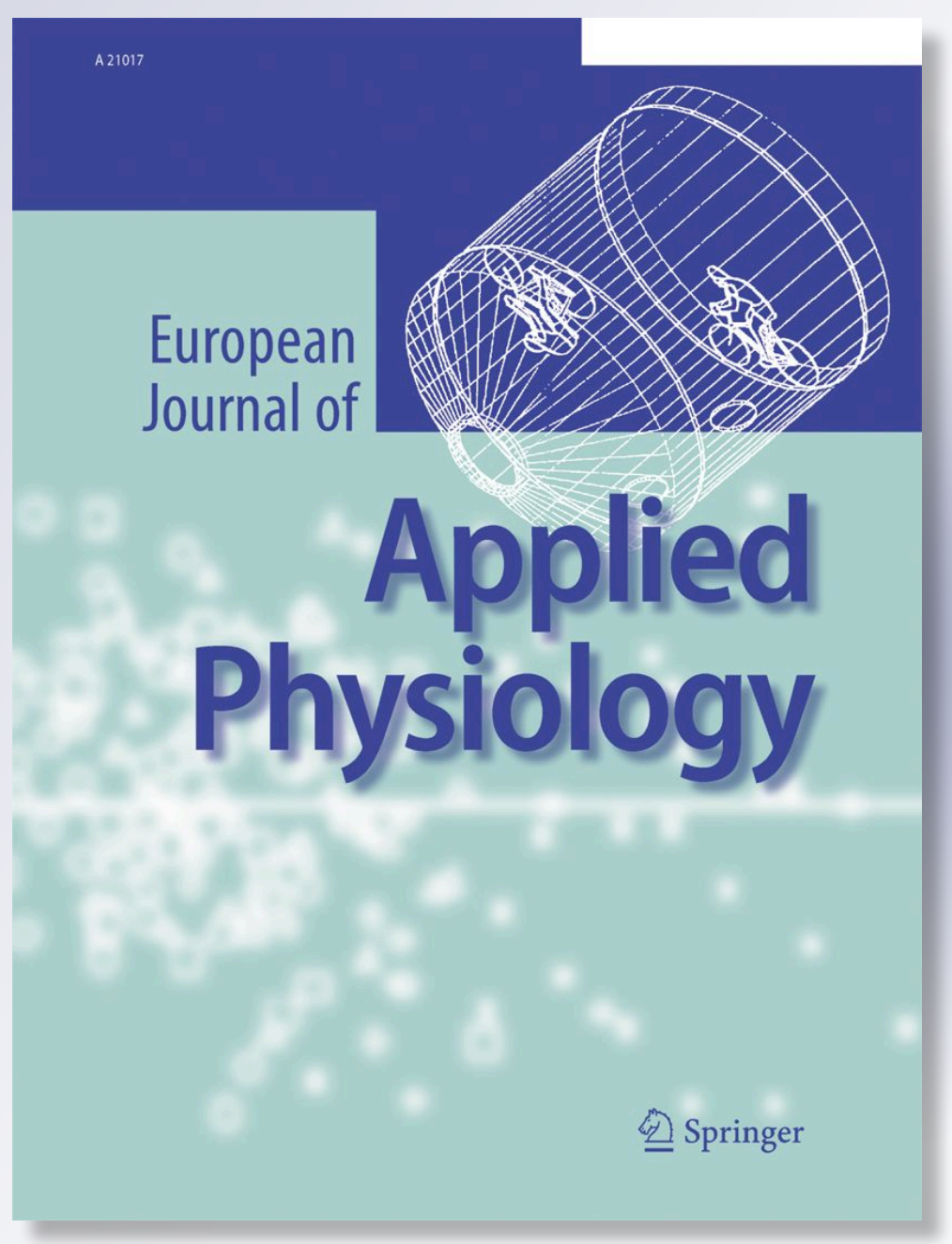

Springer 
Your article is protected by copyright and all rights are held exclusively by SpringerVerlag. This e-offprint is for personal use only and shall not be self-archived in electronic repositories. If you wish to self-archive your work, please use the accepted author's version for posting to your own website or your institution's repository. You may further deposit the accepted author's version on a funder's repository at a funder's request, provided it is not made publicly available until 12 months after publication. 


\title{
The unfolded protein response in human skeletal muscle is not involved in the onset of glucose tolerance impairment induced by a fat-rich diet
}

\author{
Louise Deldicque $\cdot$ Karen Van Proeyen • \\ Marc Francaux $\cdot$ Peter Hespel
}

Received: 2 September 2010/Accepted: 10 December 2010/Published online: 25 December 2010

(c) Springer-Verlag 2010

\begin{abstract}
Endoplasmic reticulum (ER) stress in pancreas, liver, and adipose tissue is a key event in the pathogenesis of obesity-related metabolic disease. Lipid-induced ER stress in liver and adipose tissue leads to inhibition of insulin signaling. Whether this mechanism exists in skeletal muscle is currently unknown. The present study aimed at assessing the ER stress response in skeletal muscle of subjects receiving a hyper-caloric fat-rich diet (HFD). Seven healthy males $(20.6 \pm 0.5$ years; $70.9 \pm 3.4 \mathrm{~kg})$ volunteered to participate in the study. They received a hyper-caloric $(+30 \% \mathrm{kcal})$ fat-rich $(50 \% \mathrm{kcal})$ diet for 6 weeks. An oral glucose tolerance test (OGTT) was performed, and muscle biopsies were taken before and after HFD. HFD increased body mass by $\sim 3 \mathrm{~kg}(P=0.007)$ and the sum of skinfolds by $15 \%(P=0.003)$. After HFD, blood glucose concentrations were higher during OGTT (two-way ANOVA, $P=0.023 ;+45 \%$ at $20 \mathrm{~min}$, $P=0.002)$, and fasting plasma insulin level tended to be higher $(+20 \%)$. HFD increased intramyocellular lipids content by $\sim 50$ and $75 \%$ in type I $(P=0.0009)$ and IIa fibers $(P=0.002)$, respectively. The protein expression of inositol-requiring enzyme $1 \alpha$, protein kinase R-like ER protein kinase, $\mathrm{BiP}$ and calnexin and the mRNA level of
\end{abstract}

Communicated by Håkan Westerblad.

L. Deldicque and K. Van Proeyen contributed equally as first authors.

L. Deldicque $\cdot$ M. Francaux $(\bowtie)$

Institute of Neuroscience, Université catholique de Louvain,

1, Place Pierre de Coubertin, 1348 Louvain-la-Neuve, Belgium

e-mail: marc.francaux@uclouvain.be

L. Deldicque $\cdot$ K. Van Proeyen $\cdot$ P. Hespel

Research Centre for Exercise and Health,

Department of Biomedical Kinesiology,

FaBer-K.U. Leuven, Tervuursevest 101, 3001 Louvain, Belgium spliced $\mathrm{X}$ box binding protein-1, CCAAT/enhancer binding protein homologous protein and activating transcription factor 4 were not changed after HFD. Despite the increase in body mass, subcutaneous fat deposits, and intramyocellular lipids content, ER stress markers were unchanged in skeletal muscle of subjects receiving a HFD for 6 weeks. This suggests that the onset of glucose intolerance is not related to ER stress in skeletal muscle.

Keywords ER stress $\cdot$ High-fat diet $\cdot$ Lipotoxicity $\cdot$ BiP . Intramyocellular lipids

\section{Introduction}

The endoplasmic reticulum (ER) is where folding and posttranslational modifications of proteins occur. Certain conditions, such as high lipid load, glucose deprivation, or increased synthesis of secretory proteins, disrupt ER homeostasis and lead to the accumulation of unfolded or misfolded proteins within the ER lumen (Zhang and Kaufman 2006). To cope with this, cells activate the unfolded protein response, a series of events that serve to restore ER function (Ron and Walter 2007). The unfolded protein response has three main effectors: ATF6 (activating transcription factor 6 ), IRE1 $\alpha$ (inositol-requiring enzyme 1 alpha), and PERK (protein kinase R-like ER protein kinase). In the basal (inactive) state, each of these factors associates with the protein chaperone BiP/GRP78 (binding protein/glucose regulated-protein 78), a member of the Hsp70 (heat shock protein 70) family. Upon accumulation of unfolded/misfolded proteins, ATF6, IRE1 $\alpha$, and PERK are released from BiP/GRP78 and become activated. The downstream effects of ATF6, IRE1 $\alpha$, and PERK are to induce genes, such as XBP1 (X box binding protein 1), 
CHOP (CCAAT/enhancer binding protein (C/EBP) homologous protein), and ATF4 (activating transcription factor 4), which lead to a decrease in protein synthesis and increase protein-folding capacity. When the unfolded protein response fails, apoptosis is triggered by the cleavage of pro-caspase 4 at the ER membrane, which eventually results in cell death.

In addition to apoptosis and protein degradation, ER stress can result in decreased insulin sensitivity. The decrease in insulin sensitivity is the result of two distinct processes. First, ER stress in pancreatic islets results in dysfunctional beta-cells and in immature insulin release in the blood (Wu and Kaufman 2006). Second, ER stress in peripheral insulin-sensitive tissues, such as the liver and the adipose tissue, increases JNK (c-Jun $\mathrm{N}$ terminal kinase)mediated serine phosphorylation of IRS-1 (insulin receptor-substrate 1) and thereby inhibits insulin action via decreased signaling to Akt/PKB (Sundar Rajan et al. 2007). However, it is not clear whether similar effects occur in skeletal muscle. A recent study in diabetic patients indicated that markers of the unfolded protein response were increased in cells isolated from muscle biopsies and cultured in the presence of palmitate (Peter et al. 2009), suggesting that ER stress is present in muscle in vivo. Using a high-fat-fed mice model, we recently showed that the unfolded protein response was increased in skeletal muscles, indeed (Deldicque et al. 2010a). Due to the important role of skeletal muscle in whole body metabolic regulation and the possibility that ER stress could regulate insulin resistance, here we wanted to evaluate whether, similar to our findings in mice, high-fat diet can trigger the unfolded protein response in human skeletal muscle.

\section{Research design and methods}

\section{Protocol}

Seven physically active males (20.6 \pm 0.5 years; $70.9 \pm 3.4 \mathrm{~kg}$ ) volunteered to participate in the study, which was approved by the local Ethics Committee (K.U. Leuven). All subjects were involved in regular sports and physical activity at a rate of $\sim 3.5 \mathrm{~h} /$ week and were instructed not to change their normal participation in exercise and physical activities for the full duration of the study. Subjects gave their written, informed consent after they were informed in detail of all experimental procedures and risks possibly associated with the experiments.

The subjects were enrolled in a 6-week dietary intervention program involving hyper-caloric $(+30 \% \mathrm{kcal} / \mathrm{day})$ fat-rich $(50 \pm 1 \%$ fat $)$ feeding (HFD). Before the start of the study, subjects completed a 4-day dietary record to assess their normal dietary habits. Energy intake and diet composition were analyzed using a nutritional software package (Becel 5.00, Unilever Bestfoods). On average, subjects ingested $\sim 3,000 \mathrm{kcal} / \mathrm{day}$, of which $\sim 50 \%$ in the form of carbohydrates, $\sim 35 \%$ fat, and $\sim 15 \%$ protein. Hyper-caloric $(+30 \%$ kcal/day $)$ high-fat menus were composed by a professional dietician. Energy distribution in each dietary program was $\sim 50 \%$ in the form of fat, $\sim 40 \%$ carbohydrates, and $\sim 10 \%$ protein. Subjects received supervised weekdays lunches, whereas all other meals, snacks, and drinks were provided by the investigators as individual take-home food packages.

The pretest and the posttest were organized over three separate days each. On the first day, subjects reported to the laboratory, and fat mass was estimated from the sum of 12 skinfolds. In the evening, subjects received a standardized dinner $(1,050 \mathrm{kcal}, 60 \%$ carbohydrates, $30 \%$ fat, $10 \%$ protein), after which they remained fasted until an oral glucose tolerance test (OGTT, $75 \mathrm{~g}$ in $300 \mathrm{ml}$ water) was started on the next morning. At 20-min intervals, blood samples $(50 \mu \mathrm{l})$ were collected from the earlobe and were immediately analyzed for blood glucose (Analox GM7, Analox Instruments Ltd). Following the OGTT, subjects received a standardized diet for 3 days to avoid fluctuations in IMCL (Bachmann et al. 2001). In the pretest, subjects received a well-balanced diet $(2,500-3,500 \mathrm{kcal} ; 60 \%$ carbohydrates; $25 \%$ fat; $15 \%$ protein), while during the posttest subjects continued to adhere to the HFD as prescribed by the study protocol. At each occasion, subjects reported to the laboratory between 6:00 and 10:00 a.m. after a 12-h overnight fast. After a 30-min rest, a blood sample was taken from an antecubital vein, and a percutaneous needle biopsy was taken from the right $\mathrm{m}$. vastus lateralis. All muscle samples were stored at $-80^{\circ} \mathrm{C}$ until later analysis. Subjects were instructed to avoid strenuous exercise for at least 2 days prior to the biopsies.

Lipids measurements

Lipids extracts were prepared from biopsies (Van Veldhoven and Bell 1988), followed by analysis of phospholipids (organic phosphate), and enzymatic quantification of ceramide and diacylglycerol (DAG) by means of $\gamma_{-}{ }^{32} \mathrm{P}$-ATP and E. coli dgk kinase (Van Veldhoven et al. 1992, 1995). For determination of fiber type-specific intramyocellular lipids (IMCL) content by Oil-Red-O staining, serial sections $(4 \mu \mathrm{m})$ from biopsy samples were put on uncoated glass slides as we have previously described (De Bock et al. 2005). Fiber type-specific IMCL content was expressed as arbitrary units (A.U.). 
qPCR analysis

RNA extraction and reverse transcription have been described previously (Deldicque et al. 2010b). qPCR (quantitative polymerase chain reaction) primers were designed for human spliced XBP1 (Fwd, CCGCAGCA GGTGCAGG; Rev, GAGTCAATACCGCCAGAATCCA; NM_005080), CHOP (Fwd, CTGGCTTGGCTGACTGAG GAG; Rev, CGGGCTGGGGAATGACC; NM_004083), ATF4 (Fwd, CCAACAACAGCAAGGAGGATG; Rev, GTCATCCAACGTGGTCAGAAGG; NM_001675), RPL 19 (Fwd, CGCTGTGGCAAGAAGAAGGTC; Rev, GG AATGGACCGTCACAGGC; NM_000981) and beta2-microglobulin (Fwd, ATGAGTATGCCTGCCGTGT GA; Rev, GGCATCTTCAAACCTCCATG; NM_004048). Sybr Green ${ }^{\circledR}$ real-time PCR analyses were carried out on the iQ5 Real-Time PCR Detection System (Bio-Rad). To compensate for variations in input RNA amounts and efficiency of reverse RPL19 and beta-2-microglobulin, mRNA were used as reference genes, and results were normalized to these values. These genes were chosen using the GeNorm applet according to the guidelines and theoretical framework previously described (Vandesompele et al. 2002). A value of 1 was arbitrarily assigned to the pretreatment values to which the posttreatment values were reported.

\section{Western blotting}

The procedure for western blotting has been detailed in Deldicque et al. (2010a). After the blocking step, membranes were incubated with the following antibodies $(1: 1,000)$ overnight at $4^{\circ} \mathrm{C}$ : phospho-Akt/PKB Ser473, total Akt/PKB, phospho-SAPK/JNK Thr183/Ser185, total $\mathrm{SAPK} / \mathrm{JNK}, \mathrm{I} \kappa \mathrm{B} \alpha, \mathrm{BiP}, \mathrm{IRE} 1 \alpha, \mathrm{PERK}$, calnexin, and GAPDH (glyceraldehyde-3-phosphate dehydrogenase). All antibodies were from Cell Signaling except GAPDH from Abcam. Membranes were washed in TBST and incubated for $1 \mathrm{~h}$ at room temperature with a secondary antibody conjugated to horseradish peroxidase (1:10,000). Chemiluminescence detection was carried out using an Enhanced Chemiluminescent Western blotting kit (ECL Plus) and hyperfilms. Results are reported relative to GAPDH or to the total form for phospho-Akt/PKB and phospho-SAPK/ JNK. A value of 1 was arbitrarily assigned to the pretest values to which the posttest values were reported.

Analysis of blood samples

Plasma insulin was assayed by chemiluminescence using the Siemens DPC kit and according the instructions of the manufacturer. Plasma non-esterified free fatty acids (FFA) were determined using the Wako Chemicals reagent kit.
Statistical analyses

The effect of high-fat feeding was tested by paired Student's $t$ test. For the blood glucose and insulin concentrations data during the OGTT, treatments by time interactions were evaluated using a two-way analysis of variance for repeated measures (ANOVA). When appropriate, Student-Newman-Keuls post hoc tests were applied. The significance threshold was set to $P<0.05$. The results are presented as the mean \pm SEM.

\section{Results}

Body fat and glucose tolerance

Over the 6-week HFD, body mass increased by $3 \mathrm{~kg}$ in average (Table 1; $P=0.007$ ). This mass gain was largely, if not entirely accounted for by fat accretion as evidenced by the $15 \%$ increase in sum of skinfolds $(P=0.003)$, reflecting elevated subcutaneous fat deposits. An OGTT was performed before and after the 6-week HFD to assess whole body glucose tolerance. Blood glucose concentrations were higher during OGTT after HFD (two-way ANOVA, $P=0.023 ; \quad+45 \%$ at $20 \mathrm{~min}, \quad P=0.002$; Fig. 1a), but total area under the glucose curve was not significantly changed $\quad\left(182 \pm 35 \mathrm{mmol} \mathrm{min} 1^{-1} \quad\right.$ vs

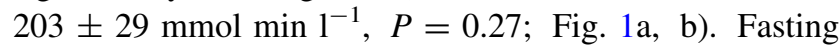
plasma insulin level tended to increase after HFD $(+20 \%$; Fig. 1a). The Matsuda insulin sensitivity index (Matsuda and DeFronzo 1999), which estimates changes in whole body insulin sensitivity, did not significantly change due to HFD $(-15 \%, P=0.28$; Fig. 1c). All together, these data indicate an onset of glucose intolerance at the end of the 6-week hyper-caloric high-fat feeding period.

Table 1 Subjects characteristics and lipid intermediates

\begin{tabular}{lcc}
\hline & Pre & \multicolumn{1}{c}{ Post } \\
\hline Body mass $(\mathrm{kg})$ & $70.9 \pm 3.4$ & $73.9 \pm 3.2^{*}$ \\
Sum skinfolds $(\mathrm{mm})$ & $134.3 \pm 27.7$ & $154.6 \pm 28.3^{*}$ \\
Plasma FFA ( $\mu \mathrm{mol} / \mathrm{l})$ & $424 \pm 42$ & $355 \pm 46$ \\
IMCL type I fibers (A.U.) & $11 \pm 1$ & $16 \pm 1^{*}$ \\
IMCL type IIa fibers (A.U.) & $5 \pm 1$ & $9 \pm 1^{*}$ \\
DAG (pmol/ng phospholipids) & $21.8 \pm 2.1$ & $21.2 \pm 2.3$ \\
Ceramide (pmol/ng phospholipids) & $6.8 \pm 0.6$ & $6.4 \pm 0.3$ \\
\hline
\end{tabular}

Body mass, sum of skinfolds, plasma FFA, IMCL, DAG, and ceramide content before (Pre) and after (Post) a 6-week hyper-caloric fatrich diet. Data provided are mean $\pm \operatorname{SEM}(n=7)$

$F F A$ free fatty acids, IMCL intramyocellular lipids, $D A G$ diacylglycerol

$* P<0.01$ versus pretest 
A

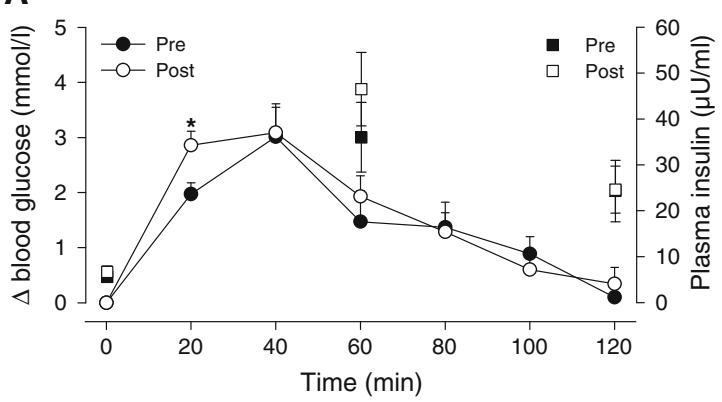

D

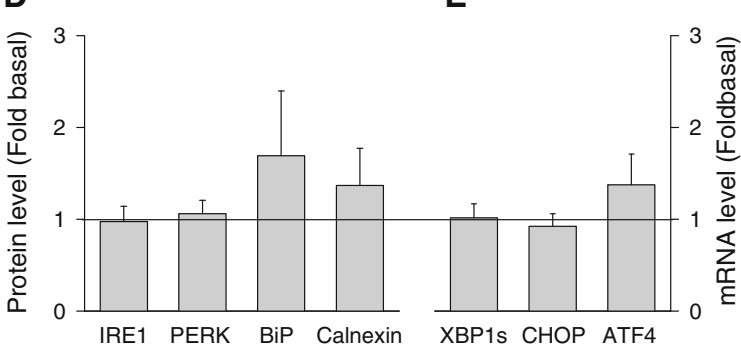

Fig. 1 Onset of whole body glucose intolerance and unfolded protein response in skeletal muscle. a Oral glucose tolerance test (OGTT, circle, left axis) and plasma insulin concentration (square, right axis) measured at 0,60 , and $120 \mathrm{~min}$ during the OGTT, $\mathbf{b}$ area under the curve $(A U C)$ and, c Matsuda insulin sensitivity index performed before (Pre) and after (Post) a 6-week hyper-caloric fat-rich diet. d Protein expression and e mRNA level of several markers of the unfolded protein response, $\mathbf{f}$ phosphorylation state of PKB and JNK,

\section{Muscle lipids}

After HFD, IMCL content was about 50 and $75 \%$ higher, in type I $(P=0.0009)$ and type IIa $(P=0.002)$ fibers, respectively (Table 1). Conversely, plasma FFA concentration, muscle diacylglycerol, ceramide, and phospholipid contents did not change between the pretest and the posttest.

\section{Unfolded protein response}

Against the background of increased body fat and IMCL content, together with onset of whole body glucose intolerance, the muscle unfolded protein response was not triggered by HFD. Neither the protein expression of IRE1 $\alpha$ $(P=0.46)$, PERK $(P=0.98)$, BiP $(P=0.54)$, and calnexin $(P=0.67)$ (Fig. 1d) nor the mRNA level of spliced XBP1 $\quad(P=0.95), \quad \mathrm{CHOP} \quad(P=0.60), \quad$ and $\mathrm{ATF} 4$ $(P=0.38)$ in skeletal muscle (Fig. 1e) were changed by HFD. The phosphorylation state of PKB was also measured to assess the basal activation of a key kinase sensitive to insulin. PKB phosphorylation is indirectly repressed by JNK via phosphorylation of insulin receptor on its serines. No difference was observed in the basal phosphorylation state of PKB $(P=0.75)$ and JNK $(P=0.89)$ between the
B

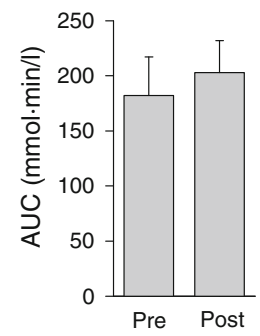

C

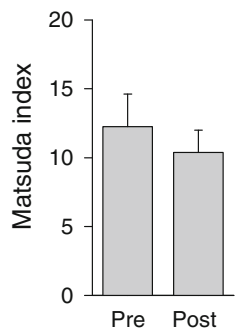

F

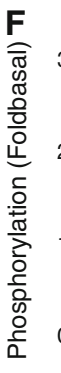

G

G

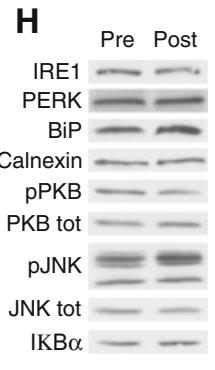

and $\mathbf{g}$ protein expression of $\mathrm{I} \kappa \mathrm{B} \alpha$ expressed as fold increase compared to the pretest. $\mathbf{h}$ Representative western blots. IRE1 inositol-requiring enzyme 1, PERK protein kinase R-like ER protein kinase proteins, $X B P 1 s$ spliced $\mathrm{X}$ box binding protein 1, CHOP CCAAT/enhancer binding protein $(\mathrm{C} / \mathrm{EBP})$ homologous protein, ATF4 activating transcription factor $4, P K B$ protein kinase $\mathrm{B}, J N K$ c-Jun NH2terminal kinase, $I \kappa B \alpha$ inhibitor of nuclear transcription factor-kappa B. $* P<0.05$ versus pretest

pretest and the posttest (Fig. 1f). The expression of $\mathrm{I} \kappa \mathrm{B} \alpha$, reflecting the activation of the NF- $\kappa \mathrm{B}$ pathway when degraded by the proteasome, was not modified by HFD (Fig. 1g; $P=0.98$ ).

\section{Discussion}

Data from studies conducted in obese rodent models have demonstrated that ER stress and the activation of related stress signaling pathways may be implicated in the pathophysiology of insulin resistance and type 2 diabetes (Gregor and Hotamisligil 2007). These data have recently been confirmed in humans as ER stress is present in adipose and liver tissues of obese people and is highly regulated by weight loss induced by gastric bypass surgery (Gregor et al. 2009). Although skeletal muscle has been much less studied than the other organs, we recently showed that the unfolded protein response is increased in skeletal muscles of mice fed a high-fat diet for 6 and 20 weeks (Deldicque et al. 2010a). The present study is the first to report data on the unfolded protein response in human skeletal muscle after a fat-rich diet. Despite an increase in body mass and subcutaneous fat deposits and onset of whole body glucose intolerance as well as an increase in IMCL content, the 
unfolded protein response was not triggered in skeletal muscle.

The rationale for choosing the present diet comes from a previous study showing that the consumption of a hyper-caloric HFD can rapidly impair glucose tolerance and insulin sensitivity in humans (Bachmann et al. 2001). In the latter study, high-fat feeding was maintained during 3 days, and the diet contained 55-60\% fats (in \% total energy intake), 30-35\% carbohydrates, $11-16 \%$ proteins and $\sim 30 \%$ more calories than the control diet. According to these previous data in healthy human (Bachmann et al. 2001), we administered a 6-week hyper-caloric HFD that was very similar with the purpose to induce glucose intolerance and insulin resistance. The choice of the 6-week duration was based on previously published results showing that a 6-week HFD in rodents increased ER stress markers in skeletal muscle (Deldicque et al. 2010a).

A recent study in diabetic patients indicated that markers of the unfolded protein response were increased in cells isolated from muscle biopsies and cultured in the presence of palmitate (Peter et al. 2009). In human myotubes, palmitate induced increased mRNA expression levels of ATF3, CHOP, and unspliced and spliced XBP1. These results suggest that ER stress could be present in skeletal muscle of diabetic patients in vivo. They also ruled out the criticism that fat cells present in muscle biopsy samples could lead to false positive results since primary myoblasts are devoid of other cell types after the isolation and plating steps. As ER stress has been shown to be involved in the pathogenesis of the metabolic syndrome, we reproduced a situation of hyper-caloric high-fat feeding, body mass gain, and fat accretion. Our data indicate that the unfolded protein response is not activated by this short-term treatment, neither is the inflammatory marker $\mathrm{I} \kappa \mathrm{B} \alpha$ nor the basal activation of insulin-sensitive kinase PKB even though fasting plasma insulin concentrations were higher. According to our data, skeletal muscle seems to face mild overnutrition (30\% more kilocalories) for a period of several weeks without showing signs of ER stress, inflammation or impairment in insulin signaling, despite the increase in IMCL.

Lipotoxicity generally involves an increase in intracellular fatty acid metabolites such as diacylglycerol and ceramides, potentially leading to ER stress, serine phosphorylation of insulin receptor via activation of JNK, and activation of nuclear factor $\mathrm{NF}-\kappa \mathrm{B}$ signaling pathways (Eizirik et al. 2008). In the present study, we did not observe any increase in JNK, decrease in basal PKB phosphorylation, or change in diacylglycerol and ceramides in skeletal muscle after a 6-week hyper-caloric highfat diet, suggesting that insulin sensitivity in skeletal muscle was not modified and that skeletal muscle did not participate significantly to the onset of whole body glucose impairment.

It is currently believed that in conditions of increased fatty acids delivery to muscle cells, like during HFD, fat oxidation is up-regulated to compensate for the overwhelming fatty acids import, which eventually may contribute to preserve insulin sensitivity (Bonen et al. 2009). Besides enhanced fatty acids oxidation, an alternative route to dispose of excess fatty acids entry into muscle during HFD is increased synthesis of triglycerides (Bachmann et al. 2001). It has been postulated that under conditions of high rates of fatty acids flux into muscle, IMCL can act as a biologically inert reservoir for fatty acids. This mechanism in turn may reduce the formation of 'toxic' lipid metabolites and protect against the development of muscular insulin resistance (Liu et al. 2007). In the current study, we found HFD to elevate IMCL content without any alteration in muscle ceramide or diacylglycerol concentrations. We did not assess fatty acid oxidation, but at the sight of the present data, it is possible that the excess fatty acids import in muscle cells during HFD which may have been compensated by the increased disposal in IMCL, thus preventing the formation of diacylglycerol and ceramide.

We showed that reduced glucose tolerance was not due to ER stress or accumulation of diacylglycerol and/or ceramide in skeletal muscle. However, based on the present data, it is difficult to identify the precise mechanism underlying the observed reduction in whole body glucose tolerance. During an OGTT, skeletal muscle and splanchnic tissues each take up 25-30\% of the glucose load (Kelley et al. 1988). Thus, we cannot not exclude that increased hepatic glucose production (Gastaldelli et al. 2000), and/or changes in gut absorption (Ley et al. 2005), rather than muscular insulin resistance largely accounted for the decreased glucose tolerance.

Due to ethical considerations, the limitation of the present study is the duration of the HFD. It is possible that ER stress, insulin sensitivity impairment and inflammation may develop in a later stage in skeletal muscle in vivo. It would be very enlightening to conduct future research in diabetic patients displaying more severe glucose intolerance.

Acknowledgments The authors thank Paul Van Veldhoven, Department Molecular Celbiology, LIPIT, K.U. Leuven, for researching data. The authors would also like to thank Raf Van Dyck, Research Centre for Exercise and Health, Department of Biomedical Kinesiology, K.U. Leuven for the assistance with the dietary analyses and Monique Ramaekers, Research Centre for Exercise and Health, Department of Biomedical Kinesiology, K.U. Leuven, for assistance during the oral glucose tolerance tests. LD has postdoctoral fellowship from the Fonds National de la Recherche Scientifique (Belgium). This study was granted by the Fonds de la Recherche Scientifique Médicale (FRSM 3.4514.) and by the Fonds Spéciaux de Recherche from the Université catholique de Louvain. This study was also 
supported by grant OT/05/53 from the Katholieke Universiteit Leuven and grant G.0233.05 F from the Fund for Scientific Research-Flanders, Belgium (F.W.O.-Vlaanderen).

Conflict of interest The authors have nothing to disclose.

\section{References}

Bachmann OP, Dahl DB, Brechtel K, Machann J, Haap M, Maier T, Lovischach M, Stumvoll M, Claussen CD, Schick F, Häring HU, Jacob S (2001) Effects of intravenous and dietary lipid challenge on intramyocellular lipid content and the relation with insulin sensitivity in humans. Diabetes 50:2579-2584

Bonen A, Holloway GP, Tandon NN, Han XX, McFarlan JT, Glatz JFC, Luiken JJFP (2009) Cardiac and skeletal muscle fatty acid transport and transporters, triacylglycerol and fatty acid oxidation in lean and zucker diabetic fatty (ZDF) rats. Am J Physiol Regul Integr Comp Physiol 297:R1202-R1212

De Bock K, Richter EA, Russell AP, Eijnde BO, Derave W, Ramaekers M, Koninckx E, Leger B, Verhaeghe J, Hespel P (2005) Exercise in the fasted state facilitates fibre type-specific intramyocellular lipid breakdown and stimulates glycogen resynthesis in humans. J Physiol 564:649-660

Deldicque L, Cani PD, Philp A, Raymackers JM, Meakin PJ, Ashford ML, Delzenne NM, Francaux M, Baar K (2010a) The unfolded protein response is activated in skeletal muscle by high-fat feeding: potential role in the down-regulation of protein synthesis. Am J Physiol Endocrinol Metab 299(5):E695-E705

Deldicque L, De Bock K, Maris M, Ramaekers M, Nielens H, Francaux M, Hespel P (2010b) Increased p70s6k phosphorylation during intake of a protein-carbohydrate drink following resistance exercise in the fasted state. Eur J Appl Physiol 108:791-800

Eizirik DL, Cardozo AK, Cnop M (2008) The role for endoplasmic reticulum stress in diabetes mellitus. Endocr Rev 29:42-61

Gastaldelli A, Baldi S, Pettiti M, Toschi E, Camastra S, Natali A, Landau BR, Ferrannini E (2000) Influence of obesity and type 2 diabetes on gluconeogenesis and glucose output in humans: a quantitative study. Diabetes 49:1367-1373

Gregor MF, Hotamisligil GS (2007) Thematic review series: Adipocyte Biology. Adipocyte stress: the endoplasmic reticulum and metabolic disease. J Lipid Res 48:1905-1914

Gregor MF, Yang L, Fabbrini E, Mohammed BS, Eagon JC, Hotamisligil GS, Klein S (2009) Endoplasmic reticulum stress is reduced in tissues of obese subjects after weight loss. Diabetes 58:693-700
Kelley D, Mitrakou A, Marsh H, Schwenk F, Benn J, Sonnenberg G, Arcangeli M, Aoki T, Sorensen J, Berger M et al (1988) Skeletal muscle glycolysis, oxidation, and storage of an oral glucose load. J Clin Invest 81:1563-1571

Ley RE, Backhed F, Turnbaugh P, Lozupone CA, Knight RD, Gordon JI (2005) Obesity alters gut microbial ecology. Proc Natl Acad Sci USA 102:11070-11075

Liu L, Zhang Y, Chen N, Shi X, Tsang B, Yu Y (2007) Upregulation of myocellular DGAT1 augments triglyceride synthesis in skeletal muscle and protects against fat-induced insulin resistance. J Clin Invest 117:1679-1689

Matsuda M, DeFronzo RA (1999) Insulin sensitivity indices obtained from oral glucose tolerance testing: comparison with the euglycemic insulin clamp. Diabetes Care 22:1462-1470

Peter A, Weigert C, Staiger H, Machicao F, Schick F, Machann J, Stefan N, Thamer C, Haring HU, Schleicher E (2009) Individual stearoyl-coa desaturase 1 expression modulates endoplasmic reticulum stress and inflammation in human myotubes and is associated with skeletal muscle lipid storage and insulin sensitivity in vivo. Diabetes 58:1757-1765

Ron D, Walter P (2007) Signal integration in the endoplasmic reticulum unfolded protein response. Nat Rev Mol Cell Biol 8:519-529

Sundar Rajan S, Srinivasan V, Balasubramanyam M, Tatu U (2007) Endoplasmic reticulum (ER) stress \& diabetes. Indian J Med Res 125:411-424

Van Veldhoven PP, Bell RM (1988) Effect of harvesting methods, growth conditions and growth phase on diacylglycerol levels in cultured human adherent cells. Biochim Biophys Acta 959:185-196

Van Veldhoven PP, Matthews TJ, Bolognesi DP, Bell RM (1992) Changes in bioactive lipids, alkylacylglycerol and ceramide, occur in HIV-infected cells. Biochem Biophys Res Commun 187:209-216

Van Veldhoven PP, Bishop WR, Yurivich DA, Bell RM (1995) Ceramide quantitation: evaluation of a mixed micellar assay using E. coli diacylglycerol kinase. Biochem Mol Biol Int 36:21-30

Vandesompele J, De Preter K, Pattyn F, Poppe B, Van Roy N, De Paepe A, Speleman F (2002) Accurate normalization of real-time quantitative RT-PCR data by geometric averaging of multiple internal control genes. Genome Biol 3:RESEARCH0034

Wu J, Kaufman RJ (2006) From acute ER stress to physiological roles of the Unfolded Protein Response. Cell Death Differ 13:374-384

Zhang K, Kaufman RJ (2006) Protein folding in the endoplasmic reticulum and the unfolded protein response. Handb Exp Pharmacol (172):69-91 